$B N-47771$

DE92 019243

\title{
ANALYSIS OF PERIODIC TRANSIENT BEAM LOADING OF THE AGS
}

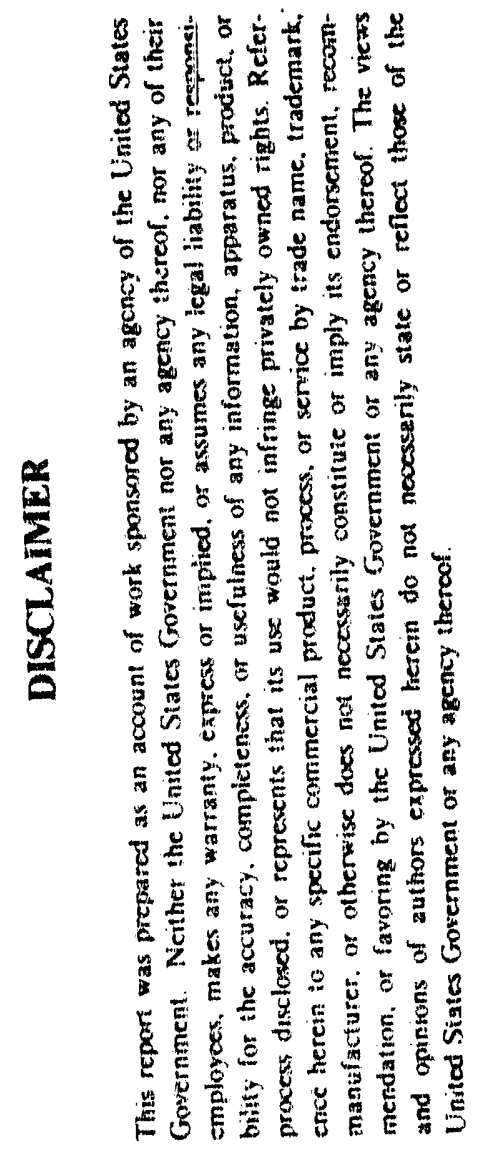

S.Y. Zhang, W.T. Weng

July 13, 1992

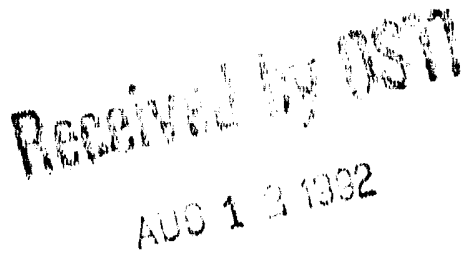

Informal. Report

Department Name

Brookhaven National Laboratory

Associated Universities, Inc.

Upton, Long Island, New York 11973

Under contract No. DE-ACO2-76CH00016 with the

UNITED STATES DEPARTMENT OF ENERGY

MASTER

1. 


\title{
ANALYSIS OF PERIODIC TRANSIENT BEAM LOADING OF THE AGS "
}

\author{
S.Y. Zhang and W.T. Weng
}

AGS Department, Brookhaven National Laboratory, Upton, New York 11873

\begin{abstract}
In this note, we discuss the multi-batch bunched bearn loading during the injection from the Booster to the AGS. The full intensity beam injection to the upgraded AGS RF system with beam phase and radial feedbacks will be studied. It is shown that a beam phase feedback is necessary in order to guarantee a predictable beam behavior after the first batch injection, otherwise the initial phase deviation for the following batch injections cannot be controlled. However, the effectiveness of the phase feedback control of the transient beam loading is limited by the associated emittance blow-up in the process. It is shown that a fast power amplifier feedback with a moderate gain can significantly reduce the transient effect of the bunched beam injection.
\end{abstract}

Work performed under the auspices of the U.S. Department of Energy 


\section{Introduction}

The operation of the AGS with the beam injection from the Booster will result in two cbanges. One is that the proton beam intensity at the AGS will be increased from $1.5 \times 10^{13}$ to $6 \times 10^{13}$ per cycle, and another is that the AGS ring will directly receive the bunched beam from the Booster. One of the possible problems is the periodic transient beam loading during the batched beam injection in to the AGS.

The AGS RF cavities and power amplifiers will be upgraded to accomnodate the increase of the beam intensity by reducing the shunt impedance and introducing fast feedback of the power amplifiers. In a perind of two years from 1992 to 1994 , however, the existing AGS RF system still bas to be used. Therefore, it is of interest to understand the performance and limitations of both the old and the new RF systems under the multi-batch beam in jection from the Booster in to the ACS.

In [1], the general static and transient beam loading effect has been discussed. In this note, we will discuss the periodic transient beam loading of the AGS during the injection. The notations and the results in $|1|$ will be followed and used in this note

The following is the beam and RF pararneters for the old and the upgraded AGS RF systems at the injection.

\begin{tabular}{|c|c|c|c|c|}
\hline \multicolumn{4}{|c|}{ AGS BEAM LOADNG PARAMETERS } \\
\hline Function & Notation & OLD & NEW & Unit \\
\hline RF Frequency & $L$ & 4.2 & 42 & $\mathrm{MHz}$ \\
\hline RF Voltage Amplitude & $V$ & 32 & 40 & $\mathrm{KV}$ \\
\hline Loaded Cavity Quality Factor & $Q$ & 21 & 19 & 1 \\
\hline Cavity Capacitance & $C$ & 82 & 175 & $\mathrm{~F}$ \\
\hline Loaded Cavity Shunt Resistance & $R$ & 9.7 & 4.2 & $\mathrm{~K} \Omega$ \\
\hline Generator Current Without Beam & $I_{\text {OQ }}$ & 3.3 & 8.52 & $\mathrm{~A}$ \\
\hline Beam Fundarnental Current & $I_{B}$ & 791 & 7.91 & $\mathrm{~A}$ \\
\hline
\end{tabular}

The AGS RF harmonic number is 12 , and there are 10 RF cavities in the AGS ring The parameters shown are for each cavity. The number of protons in one bunch, $n$, is assumed to be $0.75 \times 10^{13}$, a 50 percent larger than the designed number. We assume that 
the tuning and AVC loops will be in operation in order to guarantee a proper working col: dition for the generators and cavities. The generator current in the absence of beam, $I_{G 0}=V / R$, is important in determining the beam loading effect for a given beam current.

Two different cases will be discussed. The first is the full intensity proton beam injection to the upgraded AGS RF system, with the beam radial and phase feedbacks. The second case is also the full intensity beam injection to the upgraded system, but with a fast power amplifier feedback.

\section{Full Inteusity Beam Injection at the Upgraded AGS RF System}

The full intensity beam injection at the new AGS RF system is of interest, not only because it will be a typical operation condition in the future, but also that the resulting beam loading parameters under this condition are very close to that of the proton beam injection to the existing RF system with the intensity of $2 \times 10^{13}$. Before the fast power amplifier feedback is available, the beam phase feedback and the beam radial feedback will be utilized to control the periodic transient beam loading effect.

It takess four batch injections to fill the AGS ring. Since the cavity detuning condition will be changed from one injection to the another, each batch injection will be treated separately.

\section{First Batch Injection}

In $|1|$, the beam loading effect from the beam current to the induced beam phase deviation, $\Delta \phi$, is described by,

$$
\Delta \phi=Z_{1}(s) Z_{01} I_{B 0}
$$

where $I_{B 0}$ represents the injected beam current, which is shown in Fig.l for the periodic bearn injection at the AGS. The transfer function $z_{1}(s)$ represents the dynamic aspect of the effect,

$$
Z_{1}(s)=\frac{\sigma}{s+\sigma}
$$


where $\sigma$ is the half bandwidth of the cavity,

$$
\sigma=\frac{1}{2 R C}
$$

The scaling factor $Z_{01}$ is determined by the generator current, it can be simplified as the follows,

$$
Z_{01}=\frac{1}{I_{G 0}}
$$

under the assumption of that the stable phase $\phi_{S}$ is small, and $I_{B} \leq I_{G O}$.

Using the parameters of the upgraded AGS RF system, the beam phase deviation resulted from the frst batch injection of full proton intensity as given by equation (1) is shown in Fig. 2 over a period of $30 \mu s$.

With beam phase and radial feedbacks, the model of the beam loading is shown in Fig.3, where $\Delta R$ is the beam radius deviation. At the injection, the beam dynamic parameters are $a=1.89 \times 10^{b}$ and $b=-74$. The loop encircling $b / s, a$, and $1 / s$ represents the synchrotron ascillation, the loop encircling $Z_{1}(s), k_{1}$, and $1 / s$ is the phase feedback, and the loop encircling $Z_{1}(s), b / s, k_{2}, k_{1}$, and $1 / s$ is the radial feedback.

The beam radius deviations, which is related to the beam phase deviation $\Delta \phi$ by the transfer function $6 / s$ as shown in Fig.3, due to the first batch injection under three different conditions, namely, with the radial and phase feedbacks, with the radial feedback only, and without feedbacks are shown in Fig.4 over a period of $170 \mu \mathrm{s}$. It is shown that the radial feedback is important in controlling the heam radius deviation, and the phase feedback can further reduce the radius deviation, by providing a phase damping. Therefore, the radial and phase feedbacks are desirable. It has been shown in [2] that in general a strong phase feedback may give rise to a large and fast bucket motion, which in turn causes a large bunch motion with respect to the bucket, and hence the beam emittance blow-up. Thus, the bunch motion in the bucket must be studied, and the phase feedback gain should be chosen accordingly to minimize both radius deviation and emittance blow-up. 
Let the phase feedback gain be $k_{1}=4 \times 10^{4}$ and the radial feedback gain be $k_{1} k_{2}=4 \times 10^{4} \times 300=12 \times 10^{6}$, which will provide a radius deviation reduction, under a normal operation, by a factor of 7 , and also the phase deviation damping ratio of 0.625 . This damping ratio is low, however, it will be shown later that to increase the damping may not be acceptable due to the possibility of the emittance blow-up.

We assume that only one phase PUE and one radial PUE are used during the injection. The partly filled AGS ring provides a periodic beam phase deviation signal whose periods depend on how the ring is filled. Therefore the effective phase feedback will be varying during the injection. The gain of the radial feedback is however adjustable at the AGS according to the beam intensity, therefore the effective radial feedback can be taken as constant during the injection.

Under these conditions, the beam phase and radius deviations for the first batch injection are shown in Fig.5 and Fig.6, respectively. In a period of $30 \mu s$, the phase deviation is reduced by about 5 degrees and the radius deviation is increased to about $0.1 \mathrm{~mm}$. The bunch motion in the bucket is shown in Fig.7, which shows that the vertical bunch motion in the bucket reaches about 14 percent of the bucket height. If the phase feedback gain is increased, the damping of the phase deviation can be increased and the radius deviation can be further reduced.

\section{Second Batch Injection}

Before the second batch injection, the RF cavities have been detuned by the tuning loop. The detuned angle is,

$$
\phi_{Z 1}=\tan ^{-1}\left(\frac{I_{B 1}}{I_{G 0}} \cos \phi_{S}\right)
$$

where $I_{B 1}$ is the averaged fundamental of the beam current due to the first batch injection.

Similar to (1), the second batch injection will affect the beam phase deviation by,

$$
\Delta \phi=Z_{2}(s) Z_{02} I_{B 0}
$$


The dyamic aspect of the transfer function, $Z_{2}(s)$, is the same as the transfer function of the voltage amplitude of the detuned cavity to the beam current, which is $[3\}$,

$$
Z_{2}(s)=\frac{\sigma s+\sigma^{2}\left(1+\tan ^{2} \phi_{Z 1}\right)}{s^{2}+2 \sigma s+\sigma^{2}\left(1+\tan ^{2} \phi_{21}\right)}
$$

Since the cavity impedance with the detuning angle $\phi_{Z 1}$ becomes,

$$
Z=R \cos \phi_{Z 1} e^{-j \phi_{21}}
$$

the cavity voltage amplitude is changed by a factor of $\cos \phi_{Z 1}$ for the same beam current. The AVC loop will increase the generator current such that the amplitude of the total cayity voltage stays unchanged. Again we assume a small stable phase $\phi_{S}$, then it can be shown that if the beam current is comparable to $I_{G 0}$, the generator current will be increased from $I_{G 0}$ by a factor of $\frac{1}{\cos ^{2} \phi_{Z 1}}$. Therefore the scaling factor for the second batch injection becomes,

$$
Z_{02}=\frac{\cos ^{2} \phi_{21}}{I_{G 0}}
$$

A beam phase feedback can reduce the mean beam phase deviation of the first batch injection to zero in a relative long period of time before the second batch injection. The residual error will determine the initial condition of the beam phase deviation when the second batch enters the AGS ring.

For the sequential injection, i.e., the second batch is located adjacent to the injected batch, the beam phase deviation, the radius deviation, and the bunch motion in the bucket are shown in Figs.8, 9, 10, respectively.

For the symmetrical injection, i.e., the second batch is placed diametrically opposed to the first batch, the beam phase deviation, the radius deviation, and the bunch motion in the bucket are shown in Figs.11, 12,13, respectively.

Note that for both injections, the initial beam phase deviation is at -7 degrees, which is determined by the bearn phase deviation generated by the first batch injection, in 
existence of a phase feedback. Without phase feedback, the initial phase deviation will be large and varying, implying unpredictable beam injections following the first batch injection. Considering that beam phase deviation due to the beam loading is in a range from 20 to 25 degrees, shown in Figs. 8 and 11, if the initial phase deviation is large, then the second batch injection can cause a larger phase deviation. Therefore a phase l'eedback is needed in the periodic beam injection at the AGS.

Figs. 10 and 13 show that the bunch motion in the bucket reachs 20 to 30 percent of the bucket beight, which are larger than the motion during the first batch injection, shown in Fig.7. The effective phase feedback, which determines the motion of the bucket, depends on both the phase feedback gain and the phase deviation signal. Since the phase deviation signal becomes larger during the second batch injection, the bucket motion is larger than that during the first batch injection.

Some differences between the two injection schemes can be observed, which show that the symmetrical injection is less harmful than the sequential injection. The former generates smaller phase deviation and radius deviation, and also a smaller motion of the bunch in the bucket.

\section{Third and Fourth Batch Injections}

In the third and the fourth batch injections, the cavity detuned angle $\phi_{Z 1}$ for the second batch injection will be replaced by the larger $\phi_{Z 2}$ and $\phi_{Z 3}$, calculated from (5), and also the scaling factors will be recalculated accordingly.

For the third batch injection, the initial beam phase deviation will be different for the sequentially injected second batch, which gives rise to a -10.3 degrees of the initial beam phase deviation, and the symmetrically injected second batch, which gives rise to $a-5.4$

degrees. We show only the third batch injection after a sequential second batch injection, in Figs. 14, 15, 16, for the beam phase deviation, the radius deviation, and the bunch motion in the bucket. 
The beam phase deviation, the radius deviation, and the bunch motion in the bucket for the fourth batch injection are shown in Figs. 17, 18, and 19. The phase and radius deviations are acceptable. The bunch motion shown in Fig.19, however, indicates that the bunch

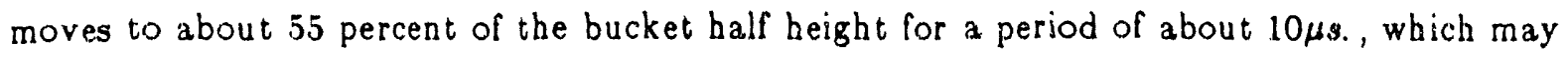
not be acceptable. Since this motion is determined by the effective phase feedback and only the phase feedback gain is adjustable, this implies that the the phase feedback gain of $4 \times 10^{4}$ is approaching the upper limit.

\section{Full Intensity Injection with Fast Power Amplifier Feedback}

The model of the beam loading effect with the fast power amplifier feedback is shown in Fig.20, where $k_{0}$ is the fast feedback gain, and $k_{1}=k_{0} \bar{k}_{1}$.

Following the same method shown above to study the beam loading effect of the periodic beam injection, with a moderate fast feedback gain of 5 , the situation is significantly improved. We show the beam phase deviation, the radius deviation, and the bunch motion in the bucket, only for the first batch injection, in Figs. 21, 22, and 23. The beam loading effect has been significantly reduced by the fast feedback.

\section{Conclusion}

In this note, we have discussed the periodic beam injection from the Booster to the AGS.

The full intensity beam injection to the upgraded AGS RF system wit heam phase and radial feedbacks have been studied. The results are useful in understanding the beam injection to the present AGS RF system with $2 \times 10^{13}$ protons per cycle. It is shown that a beam phase feedback is necessary to guarantee a predictable beam injection, because the in itial phase deviation for the second, third and fourth batch injections cannot be controlled without a phase damping. It is also shown that the phase feedback cannot provide a significant reduction to the bunched beam loading effect, because the phase feedback gain is 
limited by the possibility of a large bunch motion in the bucket, which is directly related to the beam emittance blow up.

It is shown that a fast feedback with a moderate gain can significantly reduce the impact of the bunched beam loading.

\section{Acknowledgement}

We wish to thank E. C. Raka for helpful discussions.

\section{References}

(1) S. Y. Zhang and W. T. Weng, 'Static and Transient Beam Loading of a Synchrotron,' AGS Booster Tech. Note, No. 208, BNL, July, 1992.

[2] S. Y. Zhang and W. T. Weng, 'Topics on RF Beam Control of a Synchrotron,' AGS Booster Tech. Note, No. 204, BNL, Feb. 1992.

[3] F. Pedersen, 'Beam Loading Effects in the CERN PS Booster,' IEEE Trans. On Nuclear Science, Vol.NS-22, pp. 1906-1909, 1975.

[4] D. Boussard, 'RF Power Requirements for a high Intensity Proton Collider,' Proceedings of IEEE Particle Accelerator Conference, pp. 2447-2449, San Francisco, 1991. 


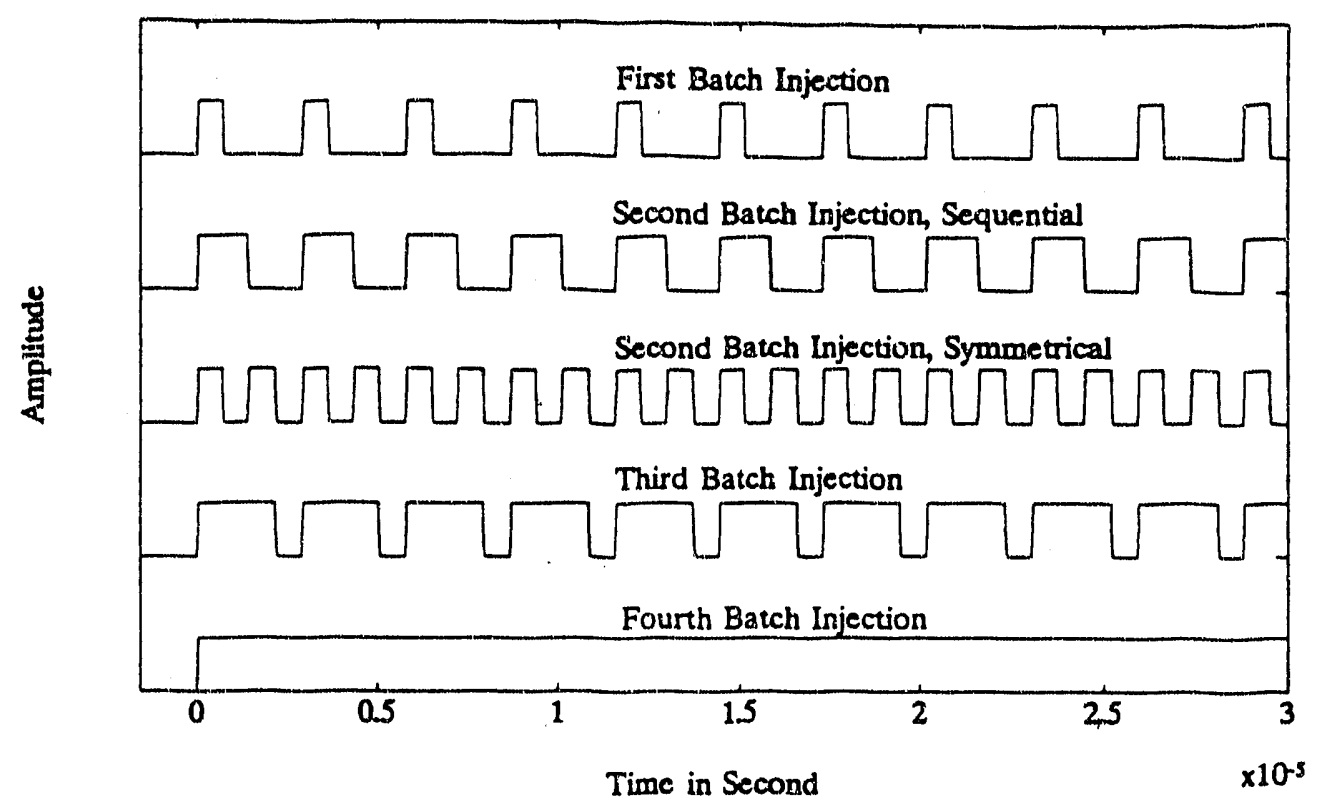

Fig.1. Waveforms of $I_{B 0}$, for Different Batch Injections.

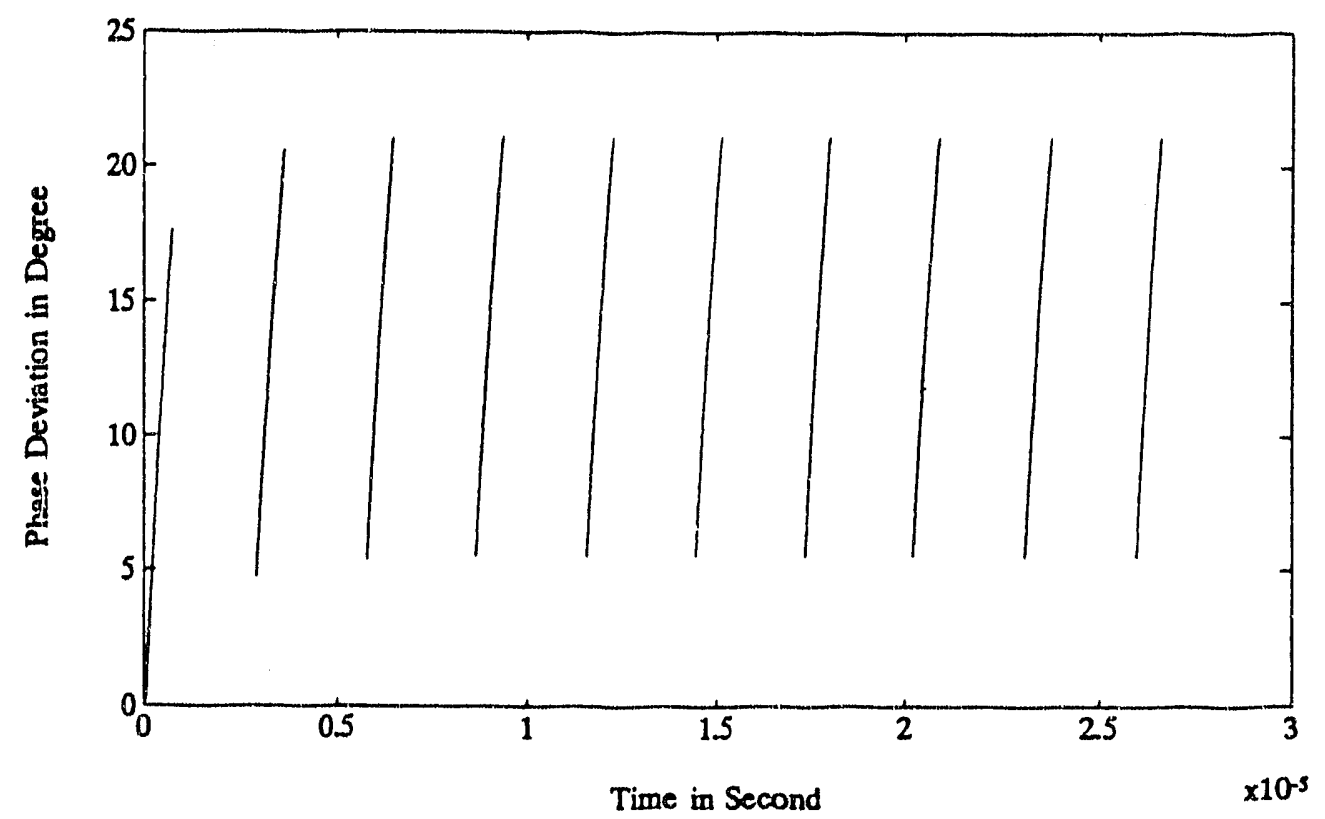

Fig.2. Beam Phase Deviation during First Batch Injection, without Feedbacks. 


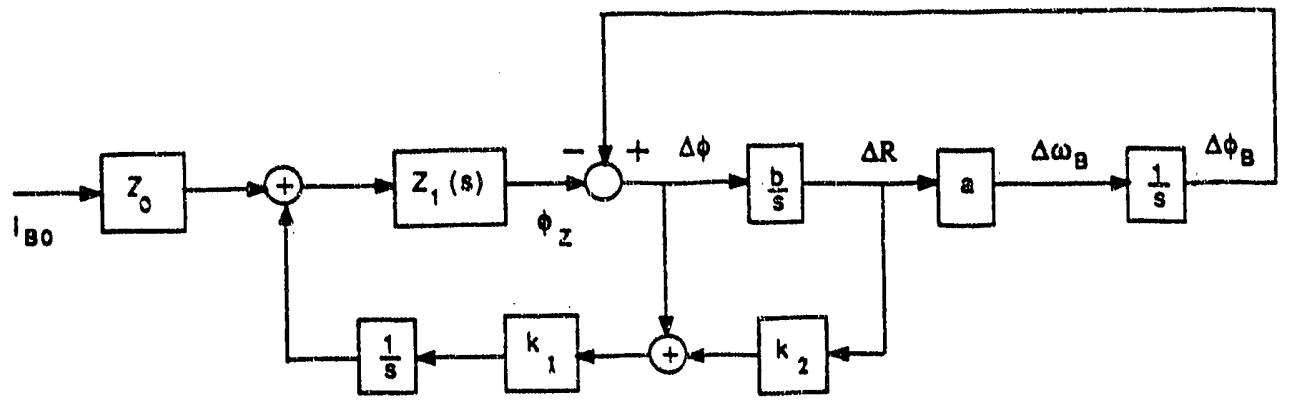

Fig.3. Model of Beam Loading, with Phase and Radial Feedbacks.

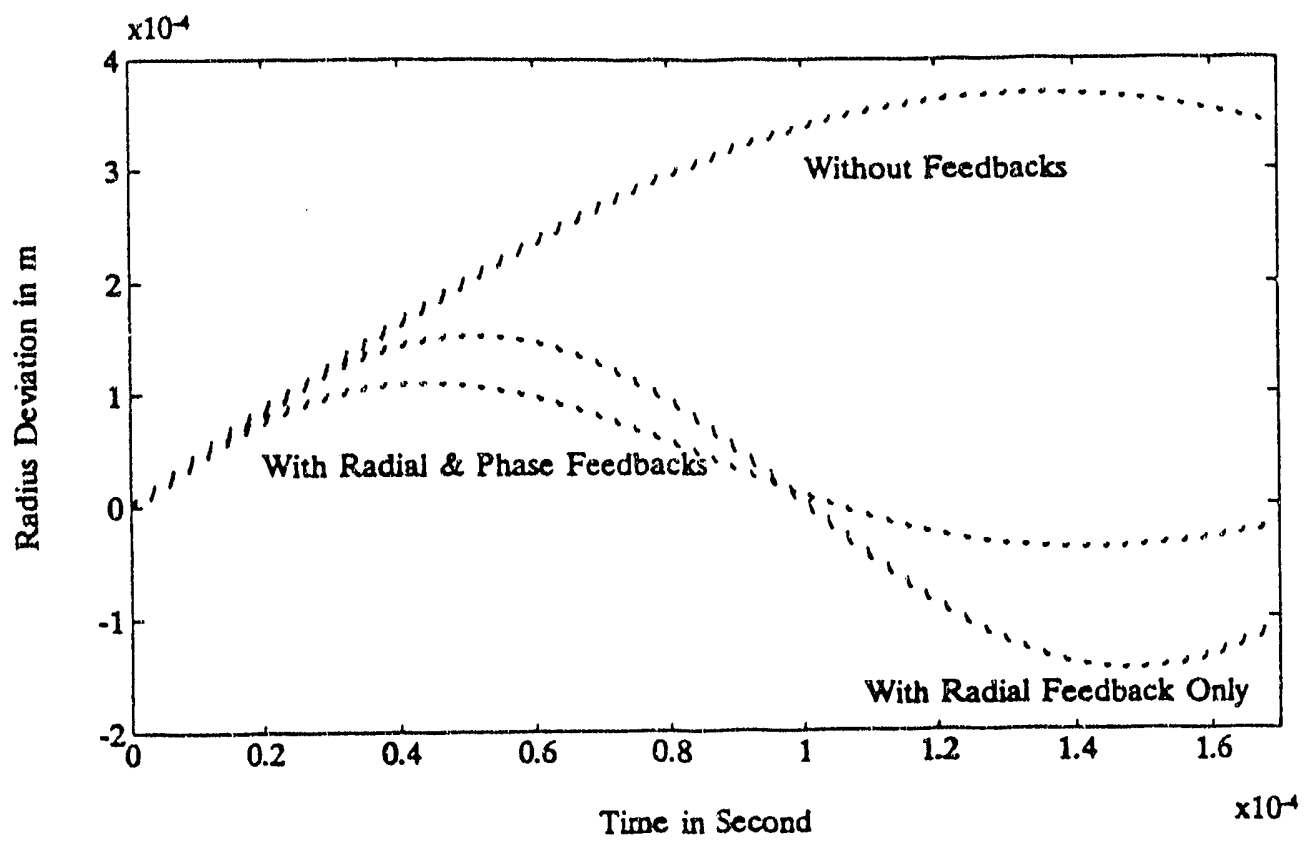

Fig.4. Comparison of Beam Radius Deviations. 


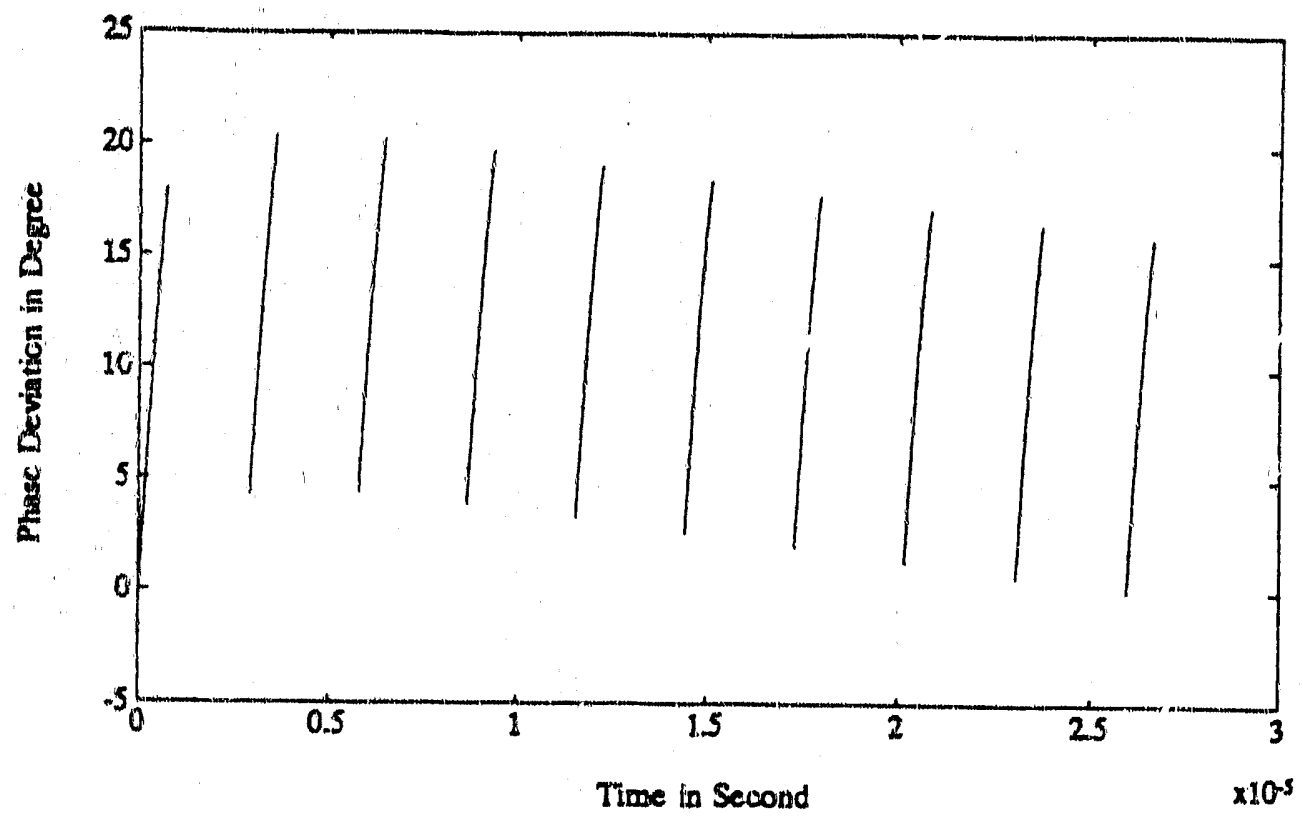

Fig.5. Beam Phase Deviation during First Batch Injection, with Phase and Radial Feedbacks.

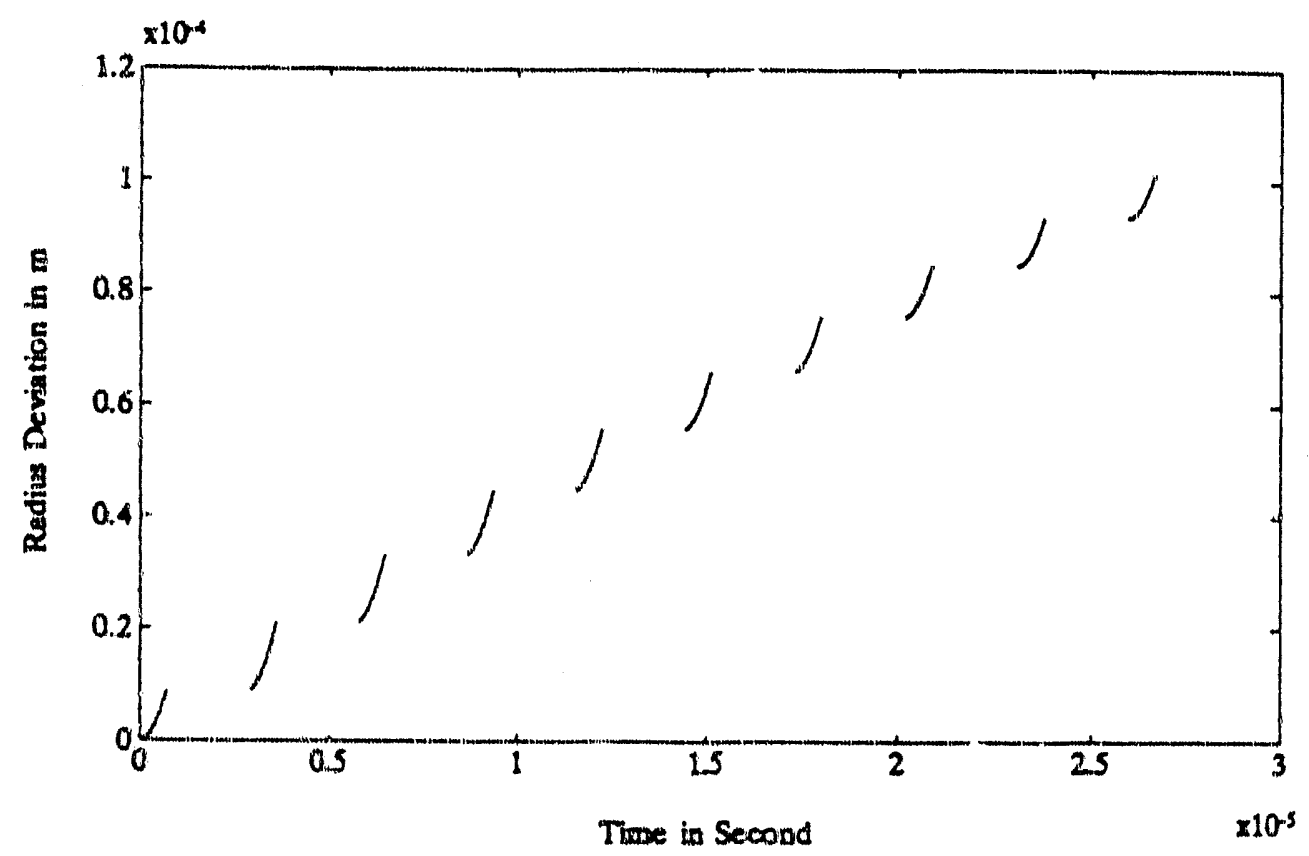

Fig.6. Beam Radius Deviation during First Batch Injection, with Phase and Radial Feedbacks. 


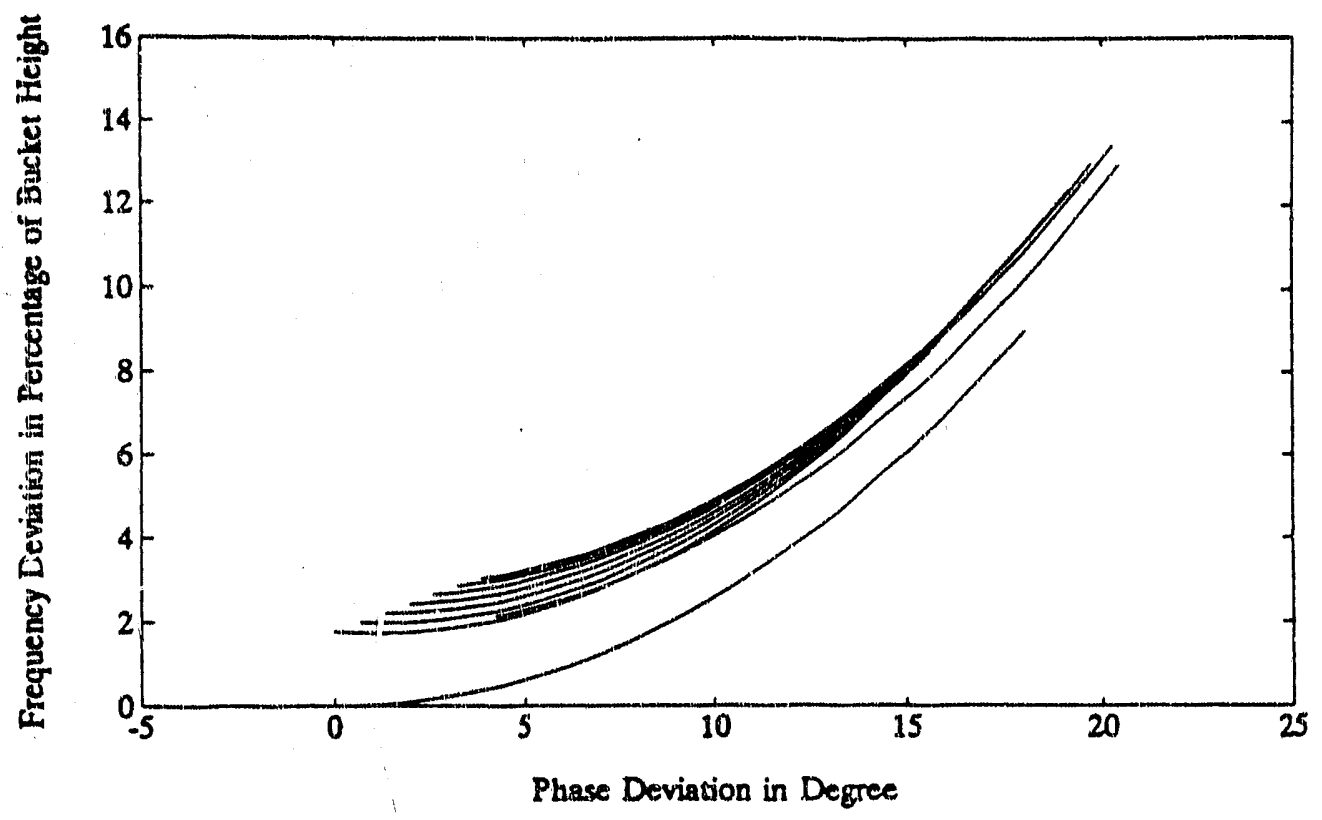

Fig.7. Bunch Motion in Bucket during First Batch Injection.

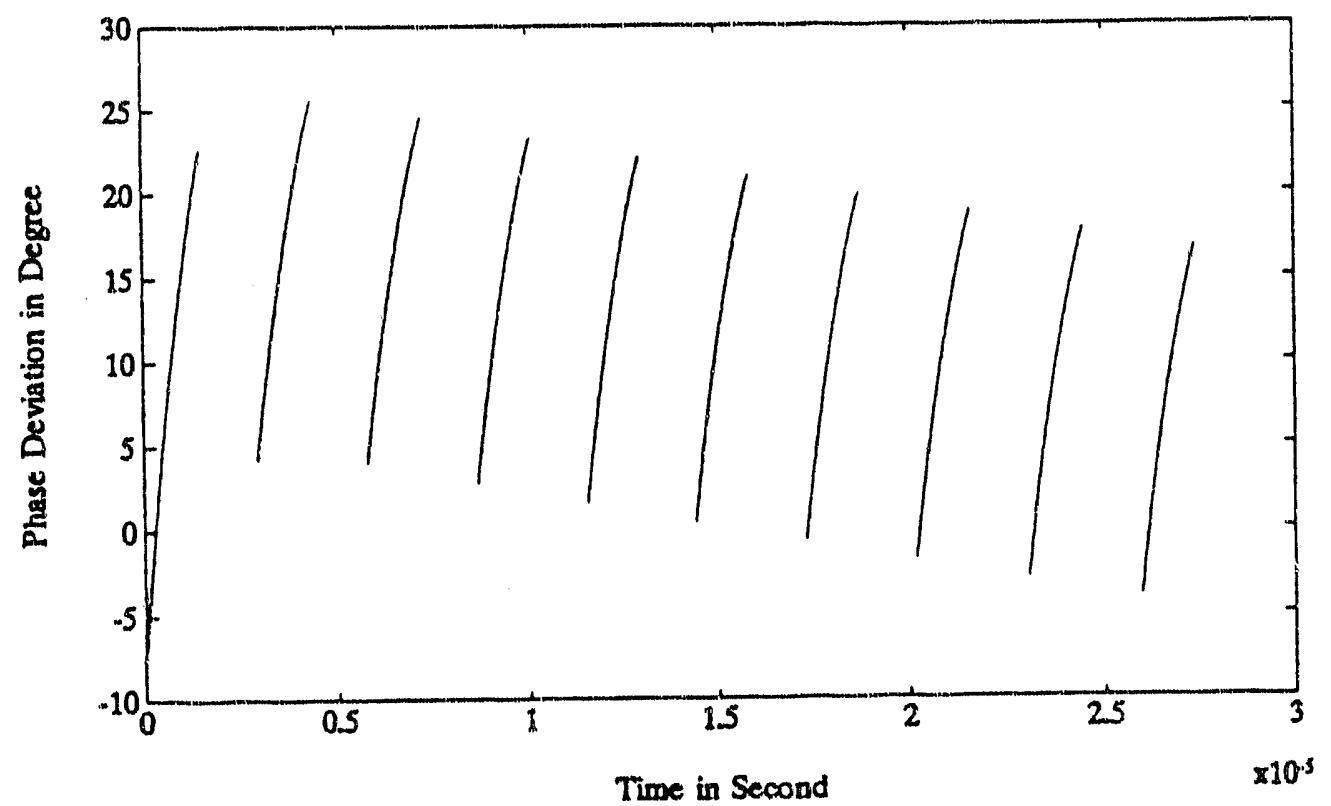

Fig.8. Beam Phase Diviation during Second Batch Sequential Injection. 


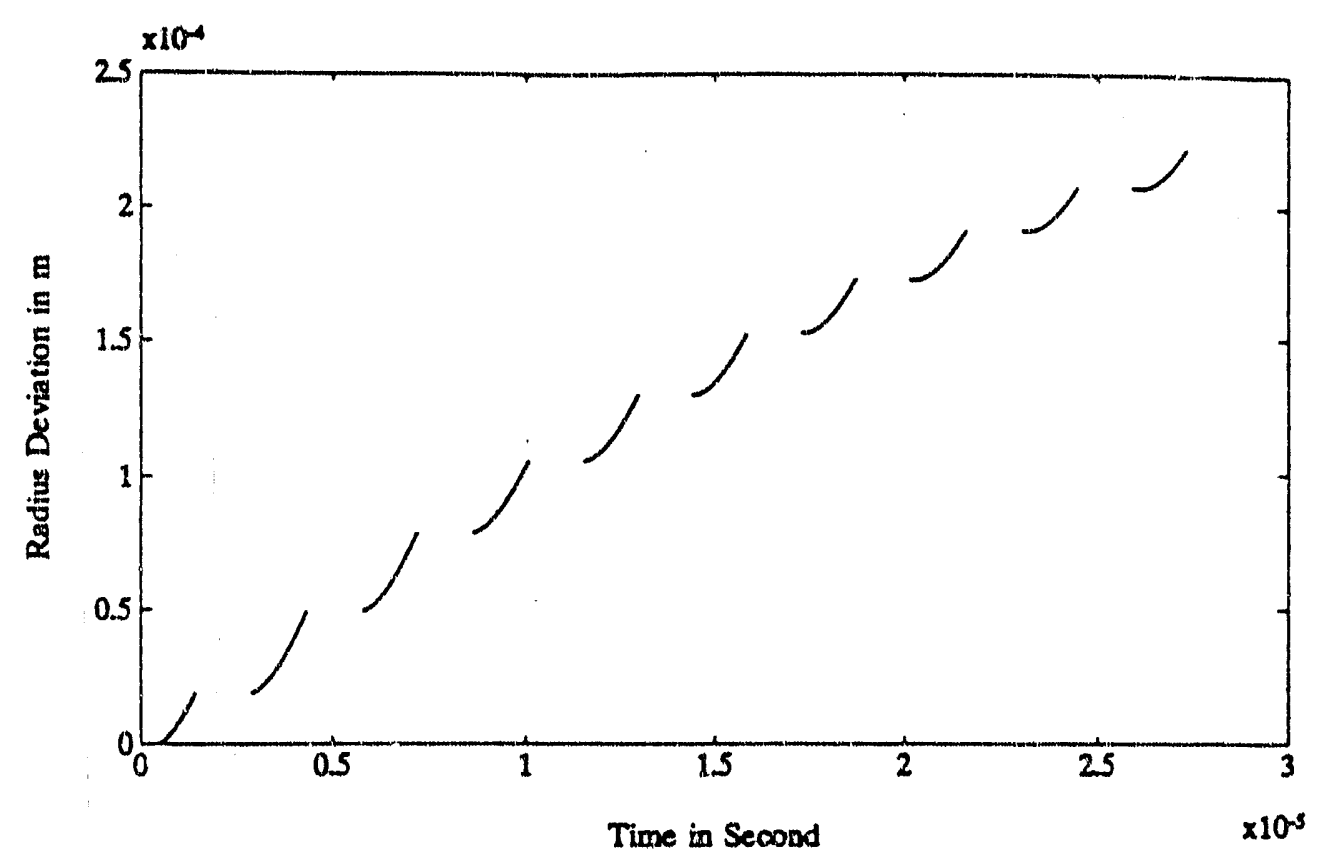

Fig.9. Beam Radius Deviation during Second Batch Sequential Injection.

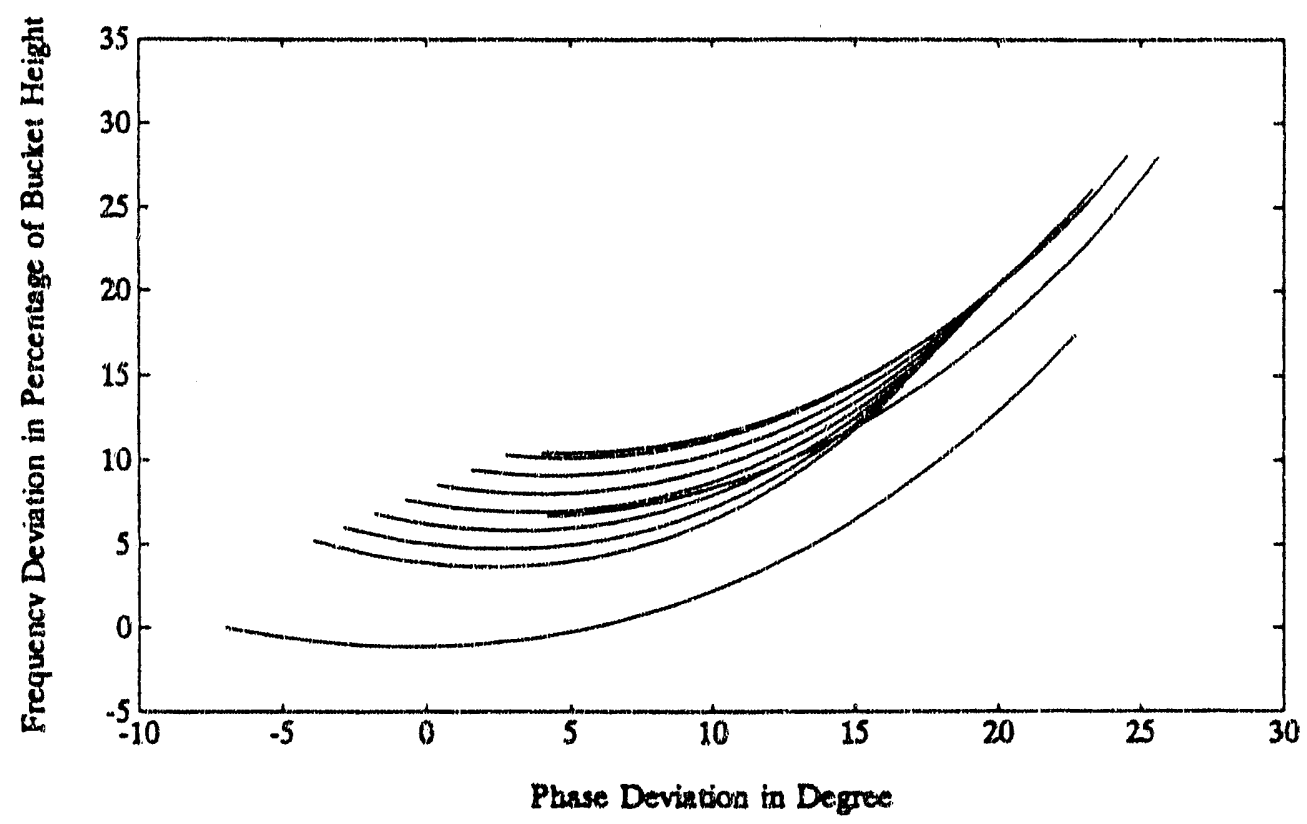

Fig.10. Bunch Motion in Bucket during Second Batch Sequential Injection. 


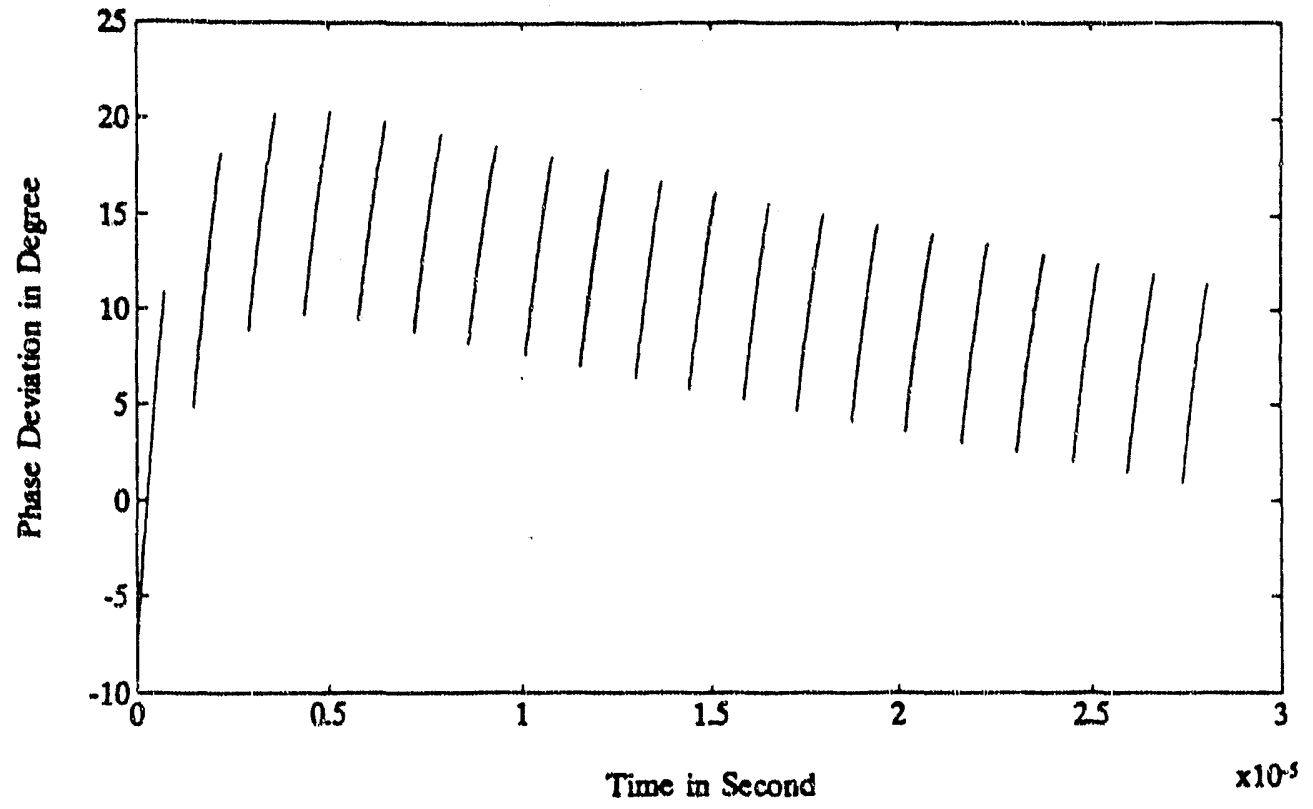

Fig.11. Beam Phase Deviation during Second Batch Symmetrical Injection.

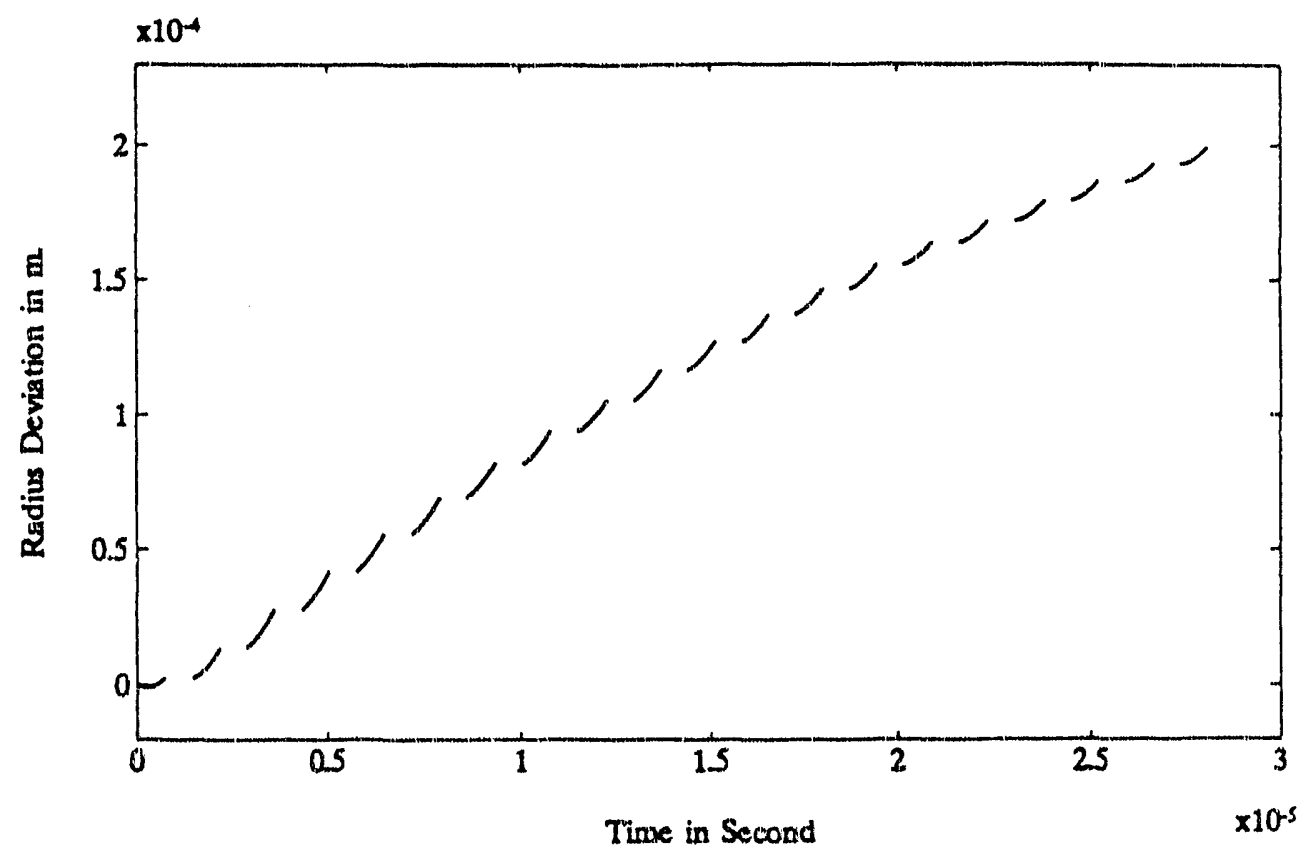

Fig.12. Beam Radius Deviation during Second Batch Symmetrical Injection. 


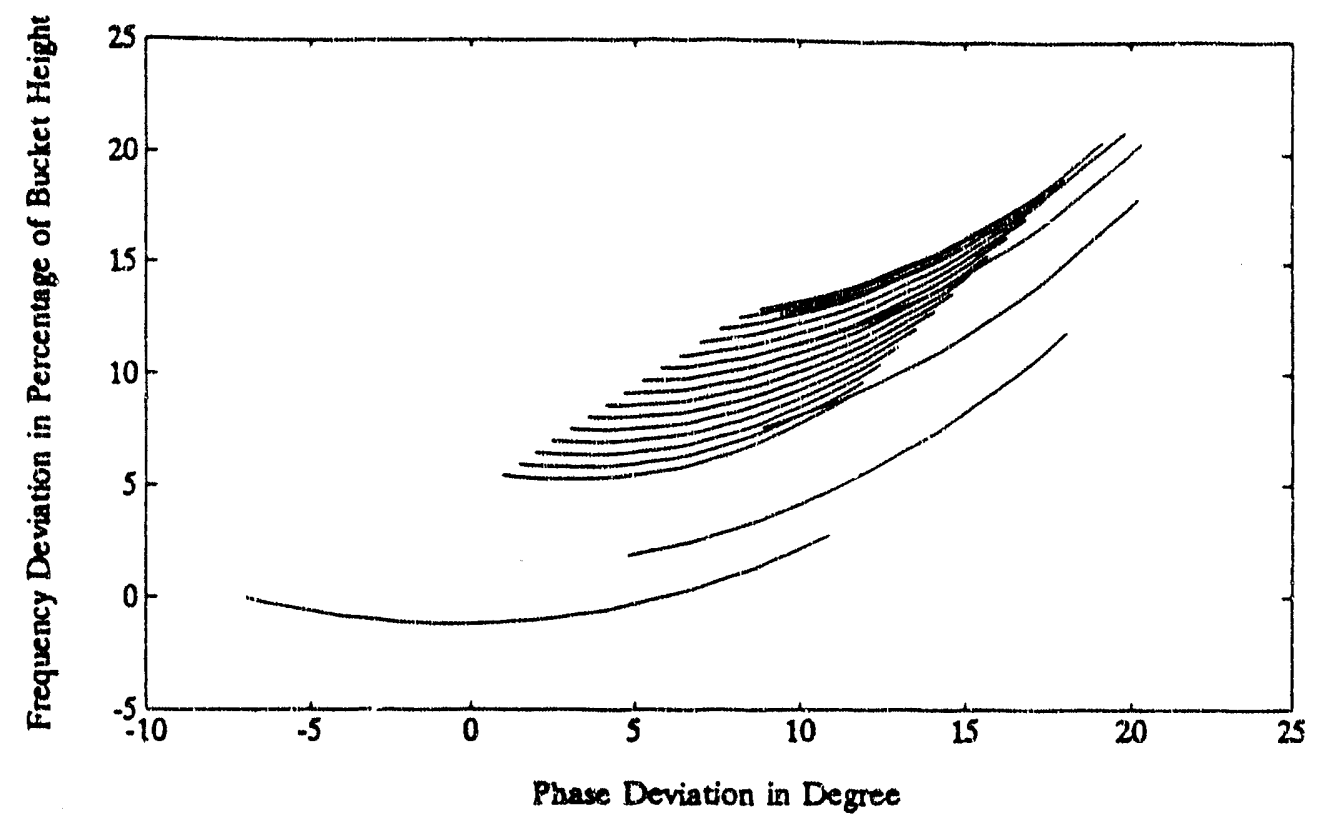

Fig.13. Bunch Motion in Bucket during Second Batch Symmetrical Injection.

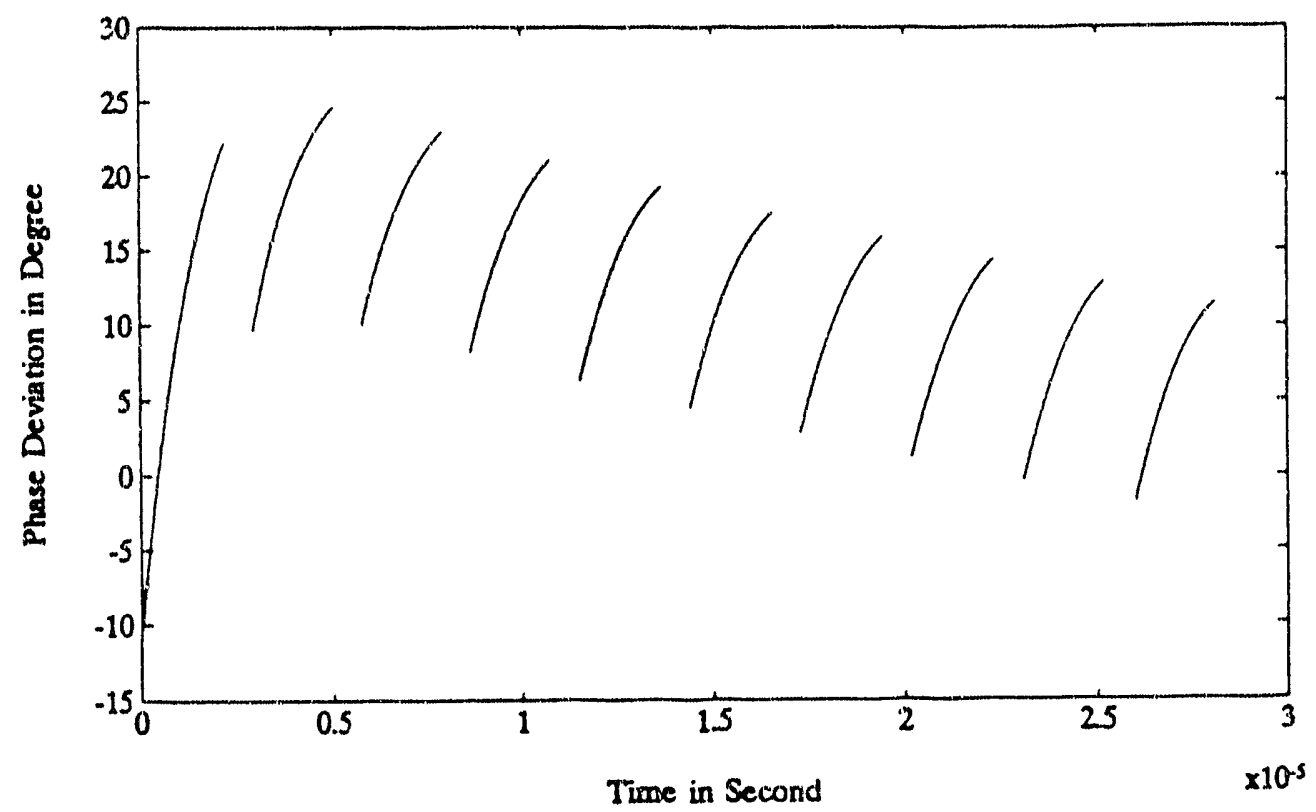

Fig.14. Beam Phase Deviation during Third Batch Injection. 


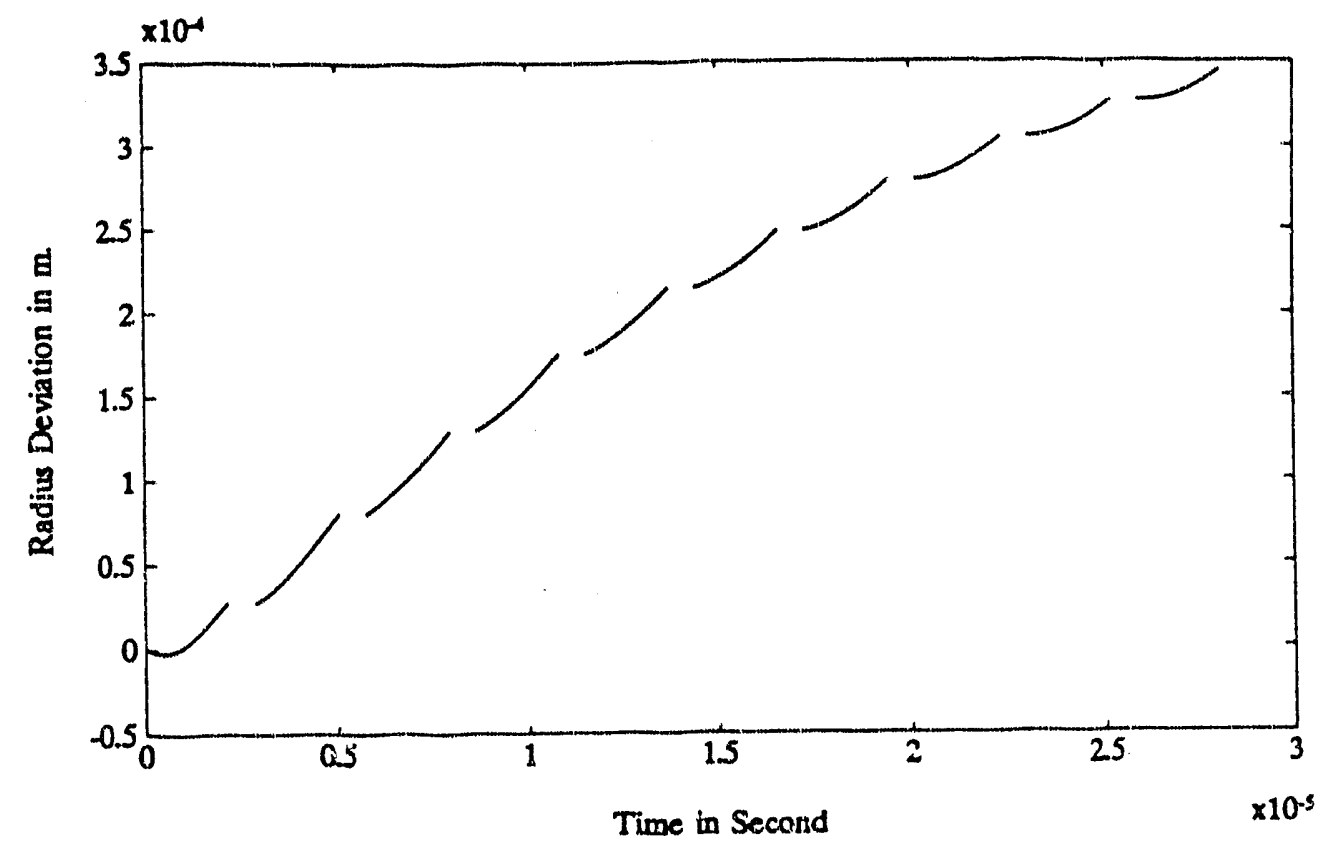

Fig.15. Beam Radius Deviation during Third Batch Injection.

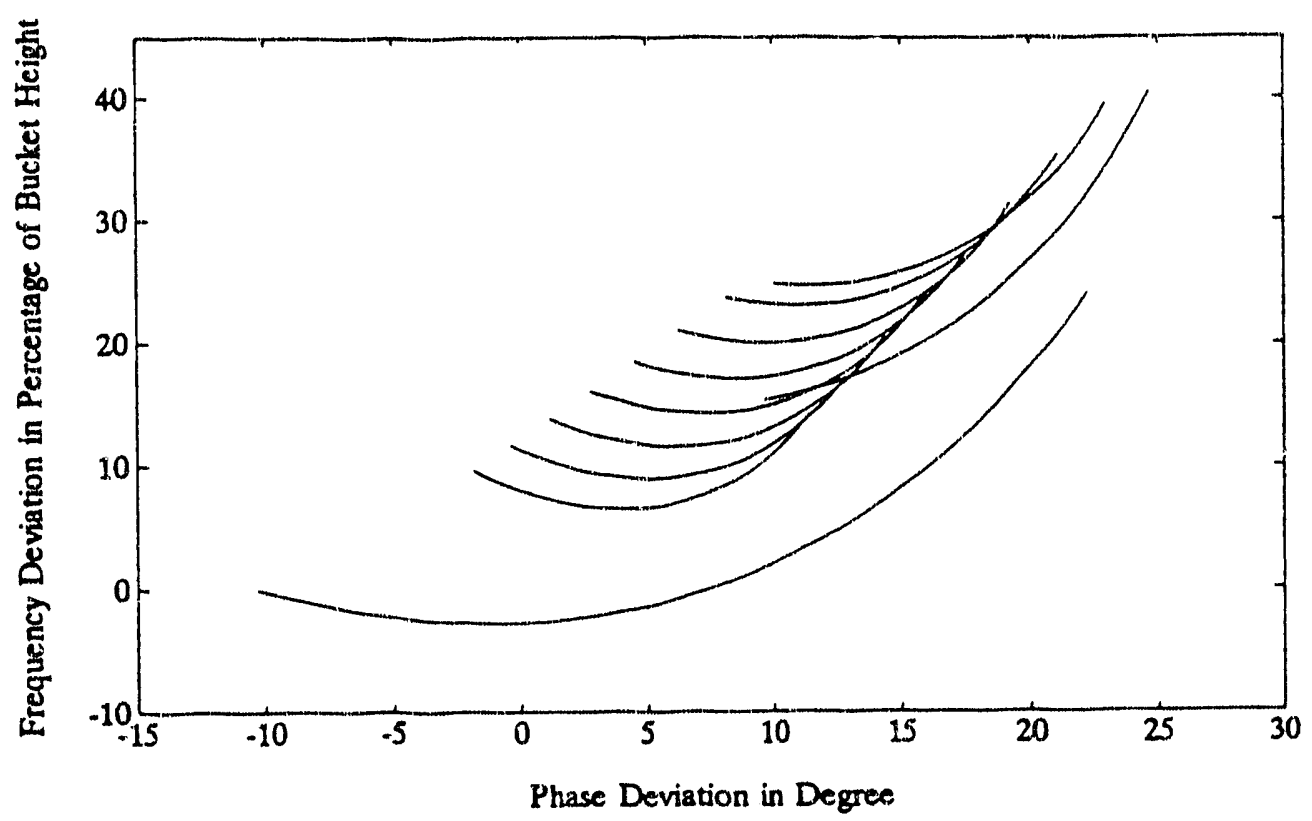

Fig. 16. Bunch Motion in Bucket during Third Batch Injection. 


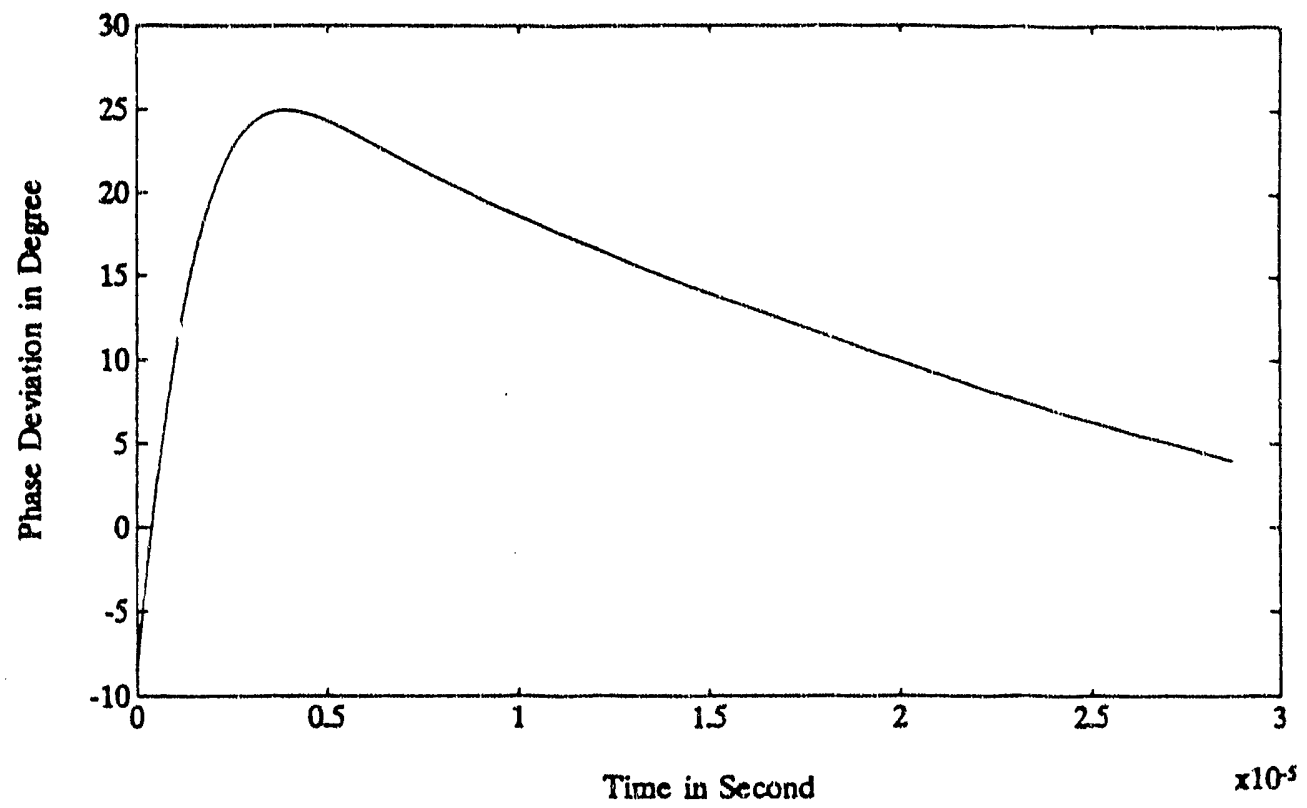

Fig.17. Beam Phase Deviation during Fourth Batch Injection.

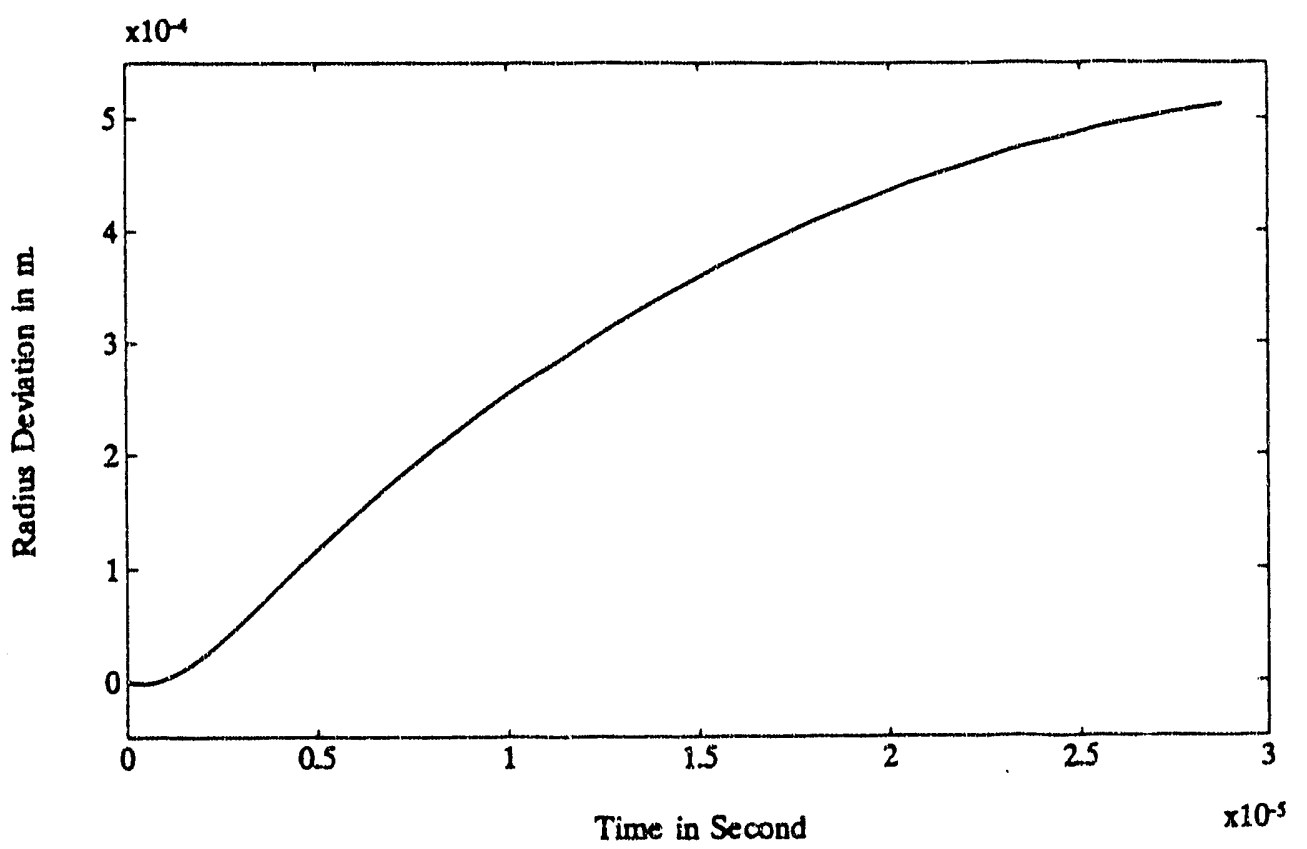

Fig.18. Beam Radius Deviation during Fourth Batch Injection. 


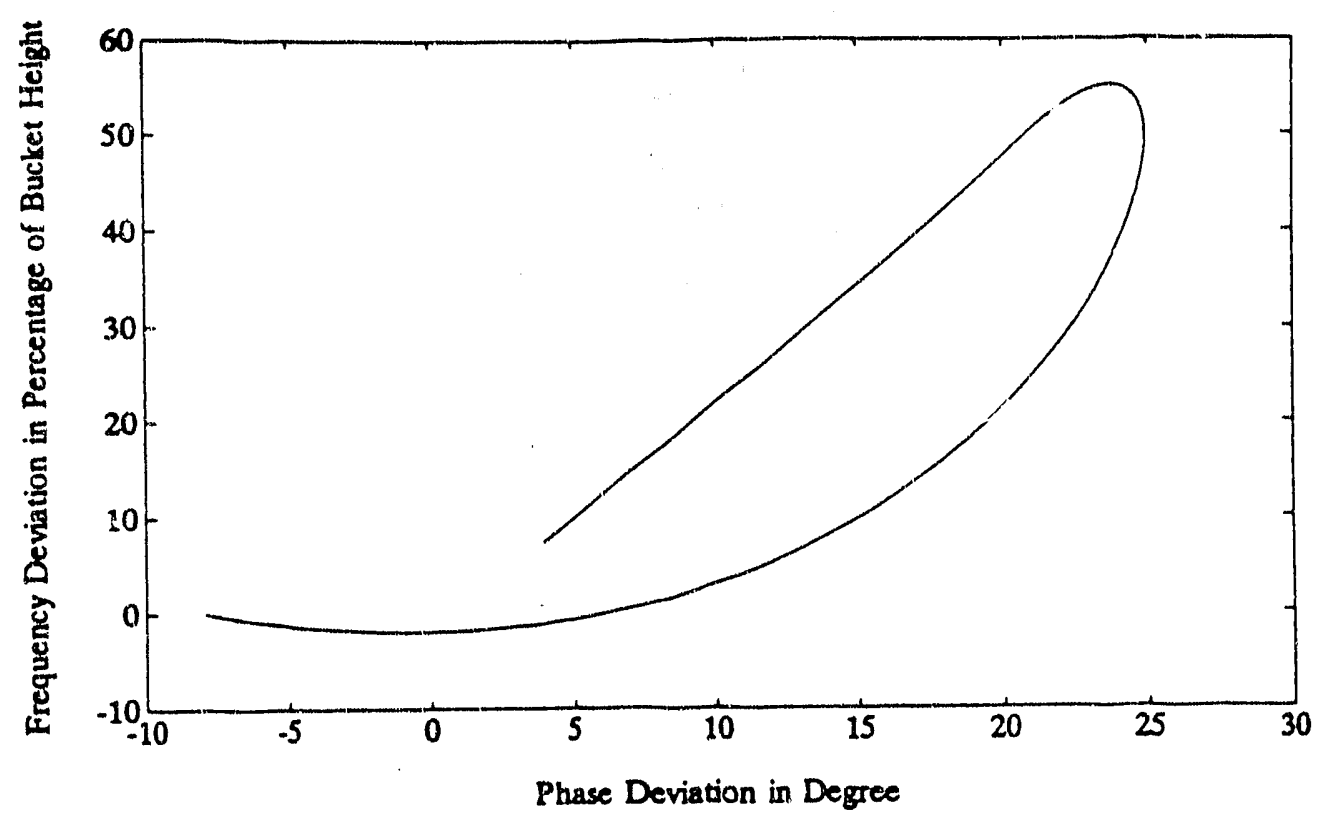

Fig.19. Bunch Motion in Bucket during Fourth Batch Injection.

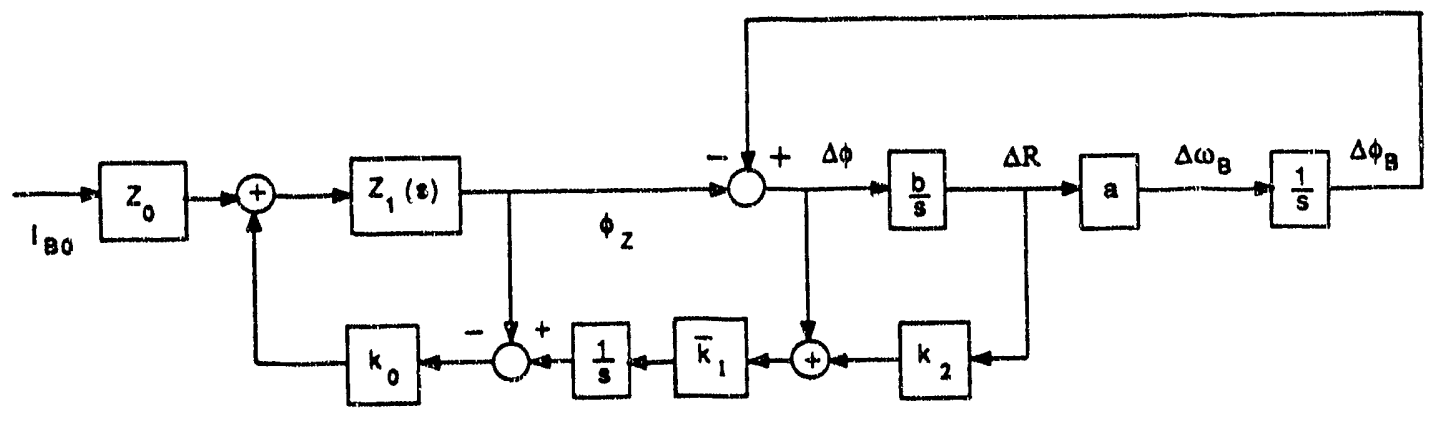

Fig.20. Model of Beam Loading, with Fast Feedback and Phase plus Radial Feedbacks. 


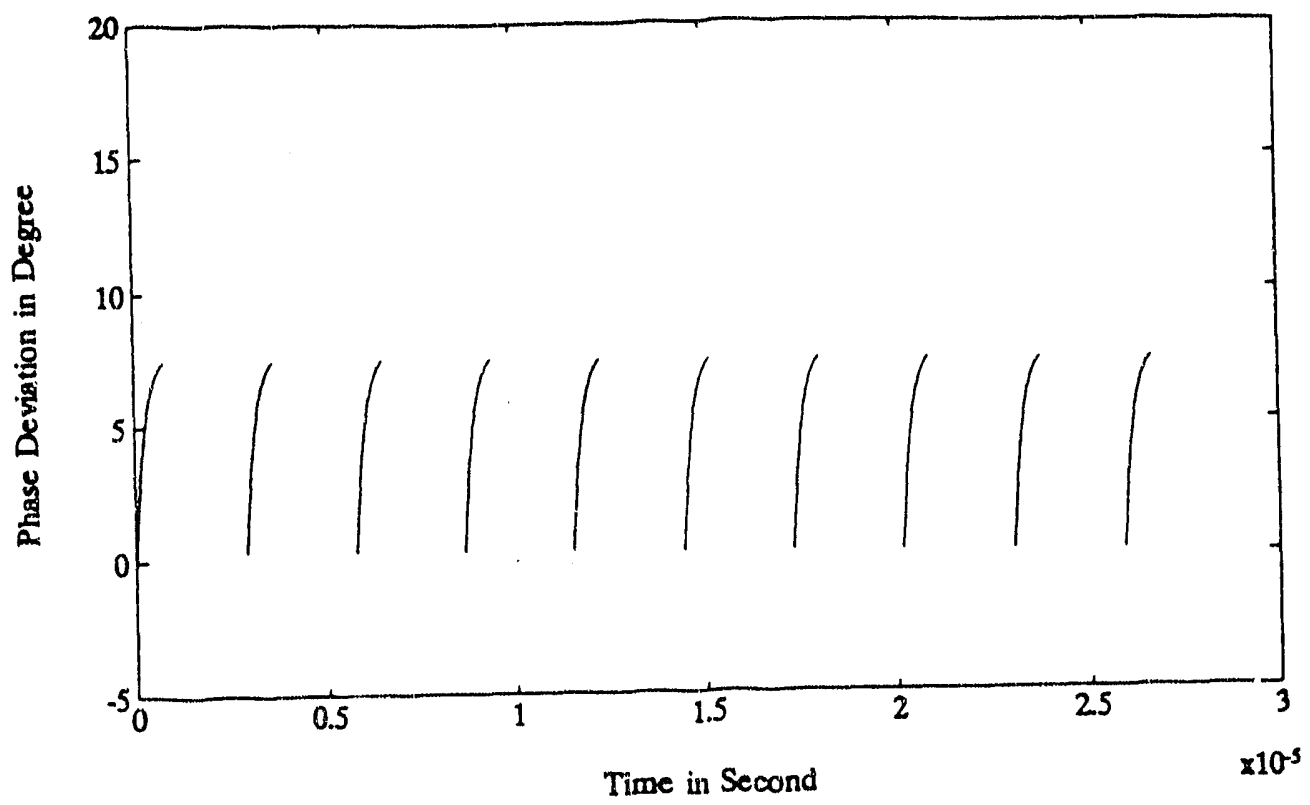

Fig.21. Beam Phase Deviation during First Batch Injection, with Fast Feedback.

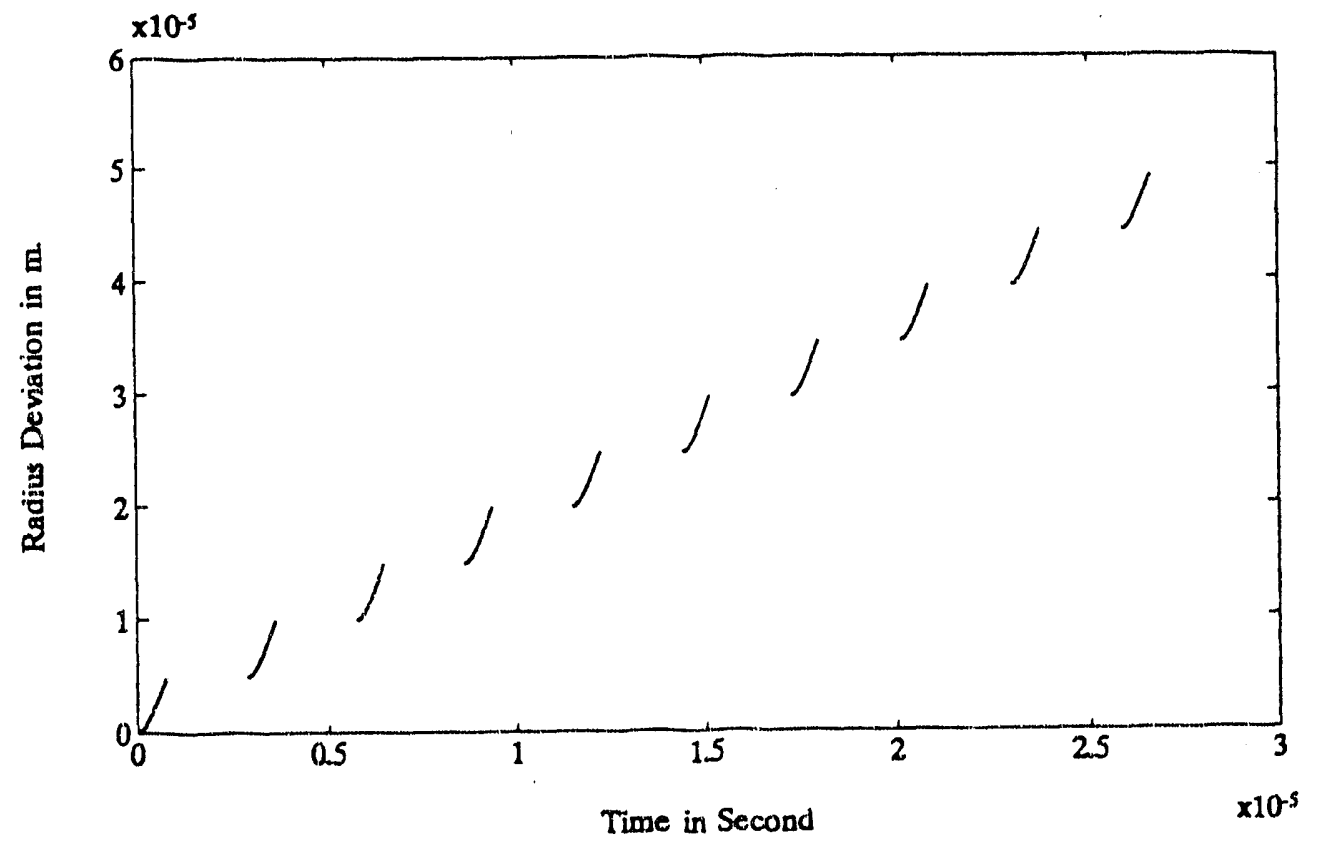

Fig.22. Beam Radius Deviation during First Batch Injection, with Fast Feedback. 


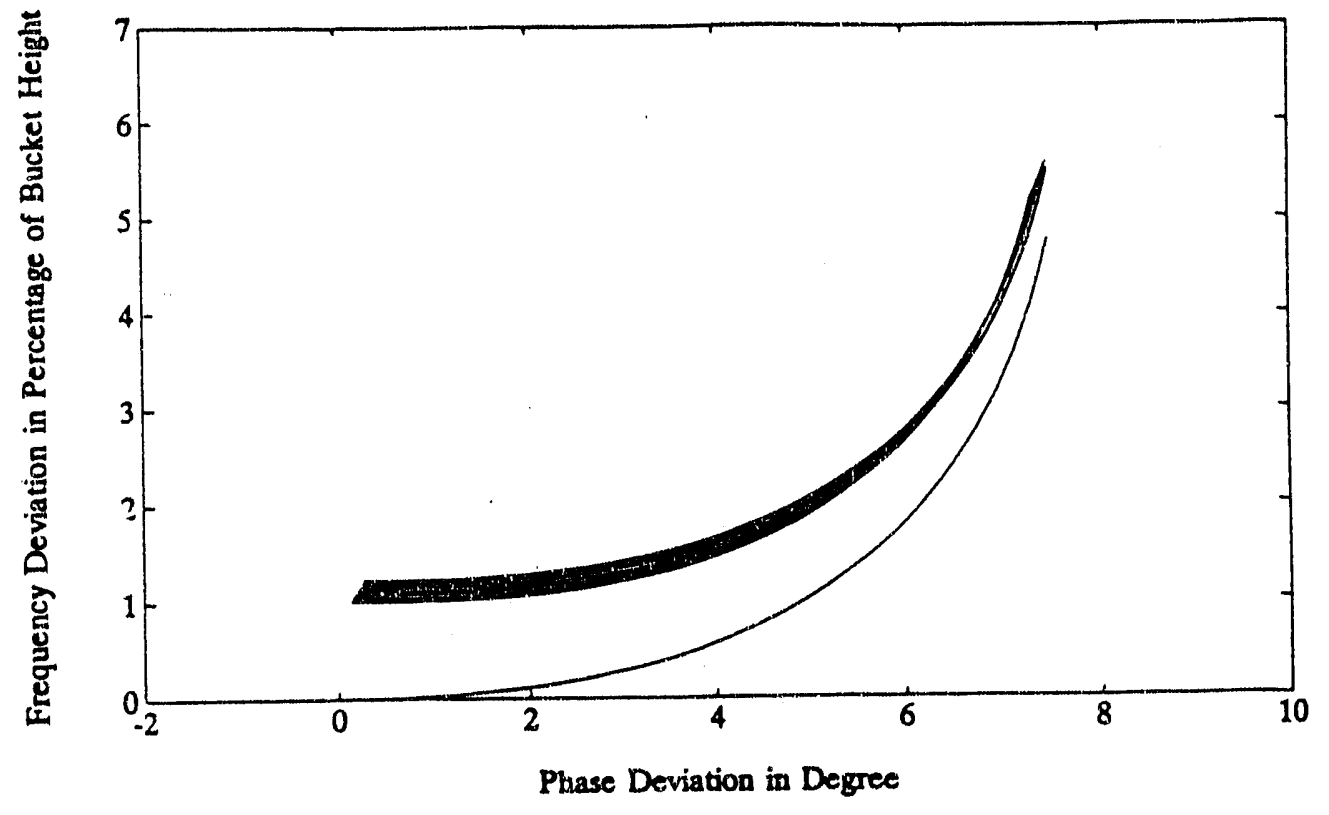

Fig.23. Bunch Motion in Bucket during First Batch Injection, with Fast Feedback. 

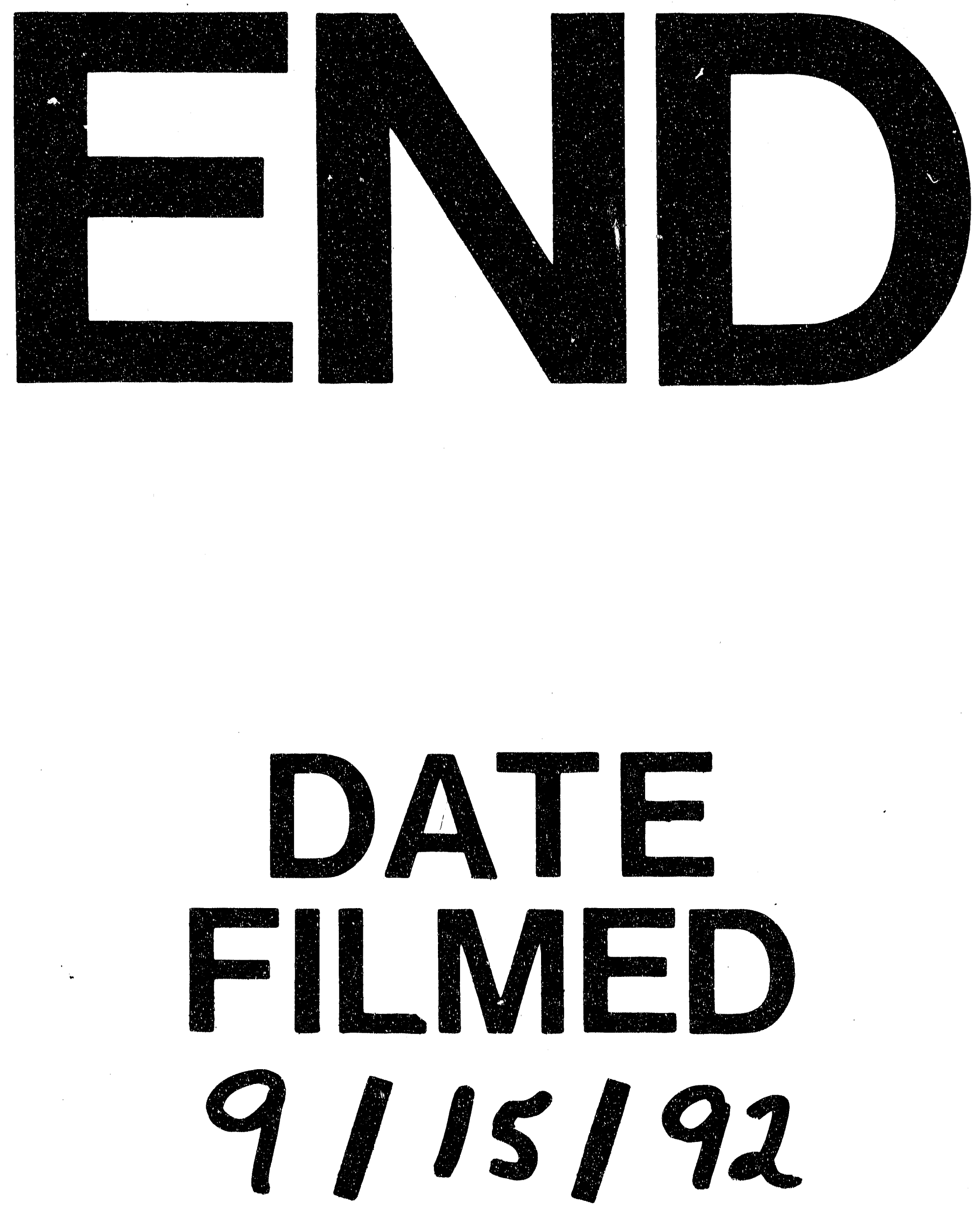
